\title{
Rethinking asset management for enterprise digital photography
}

\begin{abstract}
Aaron Holm
has been at the forefront of media technology innovation throughout his career, working in animation, audio engineering, broadcast television and digital asset management. In 2002, Aaron was recognized by the US Department of Homeland Security for his outstanding contributions in the field of interactive media technology. In 2004, Aaron founded Markham Street Media, a company dedicated to the development and deployment of advanced digital asset management systems. Working closely with Industrial Color, MSM was instrumental in the technical design and development, project management and market development for GlobalEdit, a powerful on-line image management service that is experiencing explosive growth as a pioneer in the DAM industry Since the successful collaboration, Aaron Holm has joined Industrial Color as Vice-President of Development and Integration. Industrial Color builds proprietary digital capture and asset management systems with offices in New York City, Los Angeles and Miami. The company has 25 employees and produces and manages an average of 10 shoots per day in locations across the globe.
\end{abstract}

Keywords: digital photography, digital workflow, creative group, megapixel, metadata structure

Abstract Enterprise digital imaging departments are facing massive challenges in storage and asset management with few complete solutions on the market that address their workflow needs. Photography has gone digital but the supporting workflows largely remain analog. The baseline for growth will not be set until true digital workflows are designed and built for enterprise digital photography users.

Despite the tremendous qualitative improvements in digital photography, the first thing usually done after capture is printing contact sheets and shipping them around the country. Instead, better color calibration, bandwidth, web standards, metadata flexibility, industry knowledge and realized ROI are creating a sweet spot that will allow forward thinking companies to revolutionize the medium of photography.

This paper outlines the asset management issues that enterprise digital photography departments are facing today and seeks to provide a framework for how asset management technology and techniques can be critical in helping the industry move towards a fully digital imaging workflow.

Journal of Digital Asset Management (2006) 2, 231-236. doi:10.1057/palgrave.dam.3650037

Aaron Holm Industrial Color Tel: +2123343353 Email: aholm@ industrialcolor.com

\section{OVERVIEW}

Enterprise digital imaging departments are facing massive challenges in storage and asset management with few complete solutions on the market that address their workflow needs. Photography has gone digital but the supporting workflows largely remain analog. The baseline for growth will not be set until true digital workflows are designed and built for enterprise digital photography users.

Have a look at the delegate list for the 2006 HS Digital Asset Management (DAM) Symposium in NYC and you'll get a quick sense of how important asset management is becoming to networks, ad agencies and publishers. Every major enterprise has a VP or Director level position dedicated to asset management as executives have seen the importance of managing the image lifecycle within the enterprise. This paper will focus on the pitfalls involved in managing enterprise digital photography.

Professional photography is moving steadily towards a 100 per cent digital environment. The doubts regarding quality and cost are being removed month by month -8 megapixel capture backs have become 22 megapixel backs, and are becoming 39 megapixel backs hugely improving image detail and quality. The rest of the value chain - capture, retouching and print — is working with digital files and producing quality final visuals, which are the real priority. 
So the digital workflow exists, but with major analog gaps.

\section{What's the problem?}

- Digital distribution is limited.

- Metadata structures are rigid.

- Storage is an afterthought.
Until recently there have been no real solutions to allow for the distribution of images from capture. Traditional software vendors have no incentive to transform editing into a digital process because they are focused on asset management as an end result not as an integrated workflow.

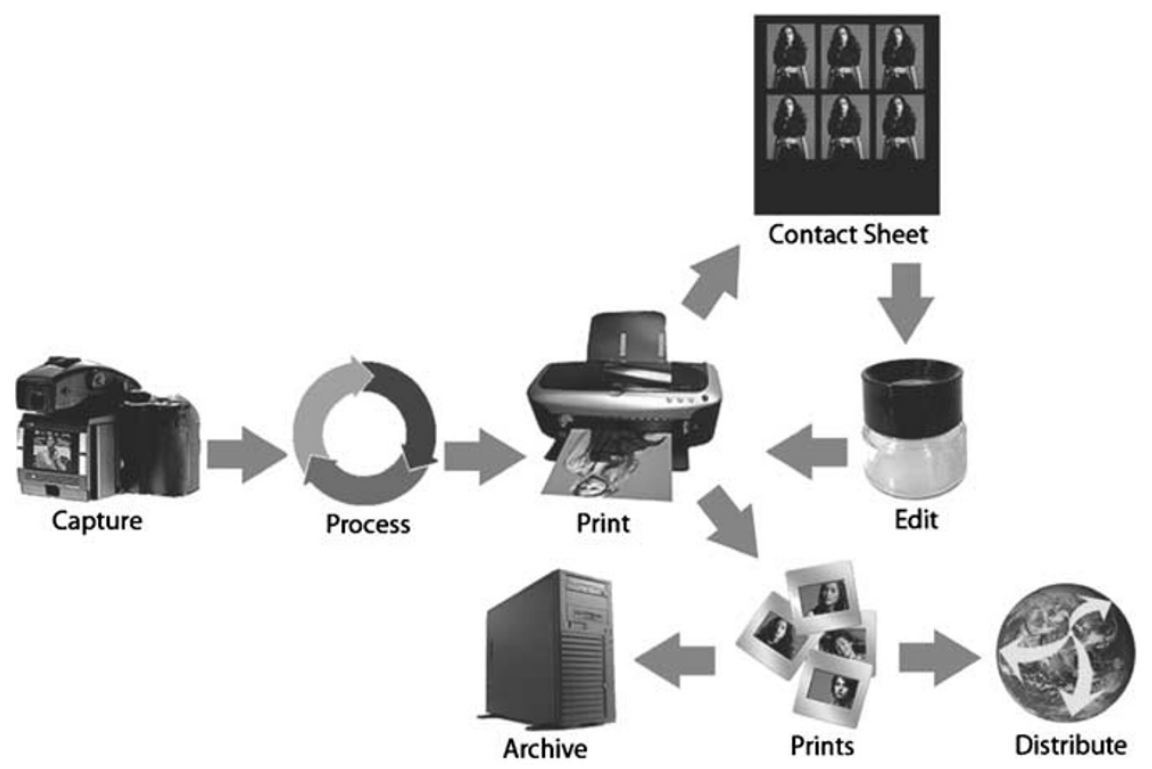

\section{DISTRIBUTION IS LIMITED - CLOSER TO THE EDIT}

A typical photo shoot day will create 2000 digital images. The Art Director and Creative group must find the images that they believe will ultimately produce a winning visual. Out of 2,000 images, only ten or 15 will survive the edit and become selects. The edit process usually consists of printing reams of contact sheets, which will be marked up by art directors, creative directors and photographers with grease pencils until a group of selects are chosen. This process has not changed since the analog days.

The promises of speed and efficiency remain trapped because the required workflow is not available in digital form. The main issues here are that:

1. It's impossible to distribute $2,00048 \mathrm{MB}$ RAW or $64 \mathrm{MB}$ processed TIFF over the internet.

2. Manually shipping drives will not enable the collaboration required in the edit.

\section{RIGID METADATA STRUCTURES}

The most basic issue presents a major challenge: how does one collect and catalog the date, location and talent info from the shoot? There are many mature methods of handling this issue including pre-configured XMP or EXIF files, stamping images with metadata during processing, or assigning staff to catalog images that return from shoot. It will always be a challenge to gather this data, but not enough attention is currently paid to understanding when the initial metadata should be captured and who should be responsible.

Also, once the initial metadata is in place, it is often impractical or difficult to change it.

The solution: design the metadata capture and edit process to individual enterprise requirements - then get it out of the way. Most people in the DAM industry view metadata as an archiving mechanism rather than part of the workflow and thus divorce it from the creative process. The accurate capture and edit of metadata throughout the image lifecycle 
is crucial, as it will drive the options available to every other component. However, we needn't treat metadata as a sacred cow that should be agonizingly detailed in its initial spec but then rigid and inflexible as our asset passes through the many hands that assign its value. Metadata should be seamlessly added and edited during every phase of our required workflow using tools that require no knowledge of metadata technology from the user. Photographers are not required to understand the structural difference between a RAW or TIFF file; why should Art Directors have to know what XMP stands for?

Additionally, metadata should be considered a component of the overall quality of the image. As much as lighting, processing and color management are crucial to enterprise quality assurance standards for imaging, so too is metadata. It's helpful to view metadata as a QA issue to ensure the topic is a priority.

\section{STORAGE - THE SCARY MATH}

All of these images do have to make it somewhere for storage. They may be in transit during production or may be reaching their final destination. This will depend on enterprise requirements and will vary from company to company but one thing remains true: most participants are not prepared for the storage requirements of enterprise digital photography. Let's go back to our sample shoot:

- A typical shoot will last 2-5 days.

- One shoot day produces an average of 2,000 shots $($ Raw $=2,000$, Jpeg $=2,000$, High Res $=100$ Tiffs; note: for entertainment $=2,000$ tiffs).

- Current professional capture backs are 22 megapixel. File size for 22MP (Raw $=48 \mathrm{MB}$, Jpeg $=2-6 \mathrm{MB}$, Tiff $=64 \mathrm{MB})$. The new generation of digital capture backs available as of January are 39 megapixel.

- Enterprise digital imaging departments often produce 50 or more shoots a year

If you don't have your calculator, that's a lot of data. That does not take into account the multiple retouched versions, layouts, proofs, etc...tack on another 10 Terabytes for these requirements. Here's where we can see the true interdependence of the solutions suggested thus far. If we have not captured the important info regarding these image files, we are storing meaningless chaos and adding time to every subsequent image related task. In addition, if the value judgments made during the edit phase were not captured electronically in metadata, we have no idea which files deserve the most attention. So, even if we've found a budget and hardware solution to store this mass of data, without an integrated workflow we don't have meaningful storage.

Taking a drive of images and dumping it onto enterprise storage is not a workflow solution. The problem with this scenario is that we have introduced an additional requirement - that the images be catalogued before they can be found and worked on by others. To get around this snag, we should build systems that ingest jobs rather than dump images. A job is a unit that is relevant to the enterprise and can be understood by everyone. Cataloging software should be replaced by ingestion workflow software that is capable of taking a meaningful collection of images and adding them to the library at the appropriate time in a single action.

There is a need to make storage meaningful. Ultimately, storage is a software, not hardware problem. Disk space is not the issue. Go to most photographer studios and you'll see the results of years of disk drive marketing. Daisy chained 500GB and Terabyte drives booting in sequence as the petrified photographer's assistant watches. The story is typical in many enterprises. People have been told that disk space is cheap, but meaningful disk space is not.

If these experienced knowledge workers actually have access to the images they need via our current generation DAM systems, they will need to pull them out, do their work, and somehow put them back without offending the rigid structure of the DAM logic container creative workers often stay with the solution that works: just make prints.

How do we begin to create solutions and truly digital workflows?

- Understand the businesses involved - advertising, entertainment, publicity and photography and design DAM solutions for creative professionals.

- Understand the technology that's available and what's coming down the pipe.

- Avoid rigid structures.

- Understanding where the analog workflow is limiting growth. 
- Find good technology and project managers.

- Find new solution providers.

What are the needs? The first need is for technology design and user requirements to find alignment. Asset management technology is typically designed for asset managers rather than the people who will do the valuable work. In this industry, the photographer, model, art directors, agencies, retouchers, publicists, talent and publishers all need to do work on an image in order for it to become valuable. In addition, each of these contributors will work with the image in a different way, using different tools.

Many of the tools required are available in software form. Products like Photoshop, and now Aperture, are industry standard and more than capable of providing a stage for creative people to work. The problem is that these software tools are not collaborative. In order to build the next generation of tools, we'll need to understand the needs of the current generation of creative professionals.

It seems unlikely that the existing crop of DAM software vendors will be able to make this leap. They have years of development investment supporting technology that exists to find digital things, not to work on them. Apple and Adobe will likely continue to lead the way in creating desktop tools for creatives, but the door is wide open on the back end.

The most important back end enabler is the internet, and the first requirement to use the internet in a digital imaging workflow is to address the issue of versioning. Through its lifecycle, an image will travel over firewire, scsi, ftp, http, Ethernet, etc... Systems built around the web must work with low-resolution images to be practical, but the retouchers and printers will ultimately require a highresolution visual. To address this, we must find ways to link these assets and provide users with the images they require, over the medium available, at the time they are needed. In addressing this need at Industrial Color we've learned that what's ultimately required is to design better interfaces, image processing systems, presentation rules, version control mechanisms and integrated storage and backup solutions. This is a significant and continuing challenge.
The good news is that the technology available to technology and project designers is ready for the challenge. More than ever before, if you can dream up a workflow, you can find capable partners to build your new generation of asset management tools.

Typical asset management tools are designed to allow users to find stuff. There's usually a group of people that work to enter information to describe a given image in order to make that image searchable within the DAM system. The problem is not the ability of existing technology to match the needs of this market, it's that the technology is deployed for the wrong group of people The Art Directors and Creatives that will ultimately work with images work with Photoshop because they were designed to meet their needs. Most asset management systems were designed to meet the needs of people looking for stuff, not people looking to do work with stuff.

Who benefits from this solution and how?

- Entertainment and Advertising Enterprises.

- Forward thinking digital photography technology and service vendors.

- Heavy users and resellers of photography and imaging. Photographers, agents and agencies will benefit from the falling price and rising quality of asset management services.

The greatest leaps are available for companies still on the fence about asset management or with systems, budgets and staff that are flexible enough to move beyond monolith systems. Entertainment companies, publishers and advertising agencies stand to gain the most as they shoot the most. Here's a breakdown of some of the benefits lurking within the new, creative workflows:

- Increased production capacity through greater efficiencies in distribution and search.

- Increased production capacity through direct integration with electronic production systems publishing, broadcast, print, pre-press, lab services, legal, HR.

- Decreased cost due to reduction in re-shoots and lost visuals.

- Decreased cost due to reduction in printing and shipping. 
- Increased client and partner satisfaction resulting from more uniform and reliable color management.

- Reduced legal expenditure resulting from formally tracked access and distribution.

- Expansion of the partner and value chain resulting from removal of geographic constraints.

- Decreased time to market for final visuals.

Asset Management executives know very well how important the economic arguments are to funding new workflow projects and changing existing processes. As the image lifecycle involves much travel, it should follow that the new workflows will involve a mix of vendor services, DAM enabled web applications, enterprise DAM at the client and, decreasingly, desktop software all working together in a well designed, defined and documented workflow.

A good example of a new beneficiary is Stockland Martel - www.stocklandmartel.com. Representing some of the world's top assignment photographers, they receive a steady stream of digital images from shoots around the world. Considering the possibilities ahead of the hazards, they have found ways to place digital at the foundation of new business opportunities. The once obscure field of asset management is of great importance to companies like Stockland Martel. Matthew Goodrich, VP of Marketing says,

"New advances and techniques in asset management have helped us run better, market better, manage better and most importantly, consider new markets and businesses."
The biggest risk in adopting new techniques is that the existing, successful processes will be destroyed. If you're a publisher who's currently operating at capacity you are doing something right. There will be no room for error as the magazine, catalog, advertisements still must go out the door. For this reason, it's absolutely essential that companies in the existing value chain step up to work with their client to improve their processes and begin to consider technology project management as a core business.

What's the good news?

- The technology required to make this work has been proven in other sectors.

- Metadata standards can connect the dots between traditional photography and advanced software development techniques.

- Distribution and search.

- New business opportunities.

Where we're all going is towards the digital workflow. Despite the tremendous qualitative improvements in digital photography, the first thing usually done after capture, is printing contact sheets and shipping them around the country. Instead, better color calibration, bandwidth, web standards, metadata flexibility, industry knowledge and realized ROI are creating a sweet spot that will allow forward thinking companies to revolutionize the medium of photography.
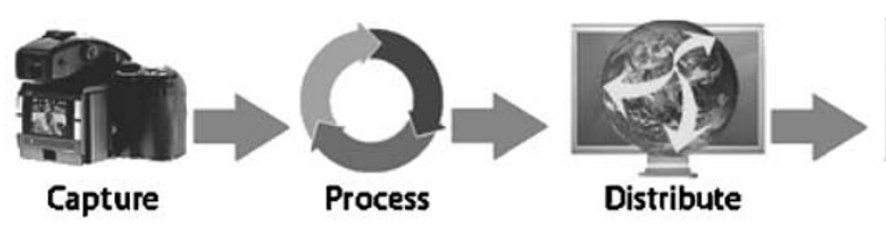

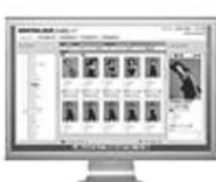

Edit

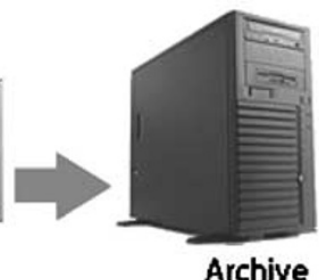

Archive
What are the risks?

- Requires heavy investment by both enterprise customers and technology service providers.

- Disrupting successful workflows in the name of innovation can lead to disaster.
The same requirements will exist in this as in previous industry changes:

- Competent managers making competent decisions in full view of the economic realities.

- Capable, experienced vendors and partners fully committed to the long-term success of every project. 
- Well-researched decisions regarding technology and consulting spending.

- Experienced project management with healthy fear of hubris.

- Careful vendor selection matching strengths with requirements.

We shouldn't have to invent any core technology here. We should thank the folks in the financial, telecom and insurance sectors for making massive investments in $\mathrm{R} \& \mathrm{D}$ that have led to the current level of sophistication available for our development purposes. Forward thinking organizations like DISC (http://www. disc-info.org) have also provided industry leadership. Storage, security, metadata, bandwidth and browser technology are more than adequate for our needs - the challenge is good user interface design. You show an art director an environment that they understand immediately and they won't care how it brings everything together, they'll just get to work. 